\title{
Prenatal Diagnosis of Suprarenal Neuroblastoma and Follow-Up After Early Surgical Treatment: Case Report andLiterature Review
}

ISSN: 2576-9200

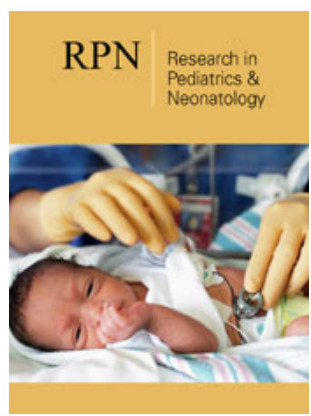

*Corresponding author: Pierre Jean Aurelus, Gastro-transplantation Department, HP CMNSXXI (IMSS), México

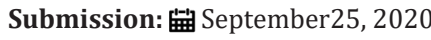

Published: 漈October 23, 2020

Volume 5 - Issue 1

How to cite this article: Pierre Jean Aurelus*, Zapata Carrión Rafael Roberto, Hermilo De La Cruz Yáñez, Ignacio Reyes Mora, Dianey Flores Flores, et al. Prenatal Diagnosis of Suprarenal Neuroblastoma and Follow-Up After Early Surgical Treatment: Case Report andLiterature Review. Research in Pediatrics \& Neonatology. 5(1). RPN. 000601. 2020. DOI: $10.31031 / R P N .2020 .05 .000601$

Copyright $(\subset$ Pierre Jean Aurelus. This article is distributed under the terms of the Creative Commons Attribution 4.0 International License, which permits unrestricted use and redistribution provided that the original author and source are credited.
Pierre Jean Aurelus ${ }^{1}$, Zapata Carrión Rafael Roberto ${ }^{1}$, Hermilo De La Cruz Yáñez $^{1}$, Ignacio Reyes Mora ${ }^{2}$, Dianey Flores Flores ${ }^{2}$, Jesús Macias Duvignau ${ }^{3}$, Roberto Carlos Manríquez Angulo ${ }^{3}$ and María Del Sol Granados Alonso ${ }^{4}$

${ }^{1}$ Gastro-transplantation Department, HP CMNSXXI (IMSS), México

${ }^{2}$ Pediatric Neonatology Department, San Angel Inn Chapultepec Hospital, México

${ }^{3}$ Obstetric and Gynecology Department San Angel Inn Chapultepec Hospital, México

${ }^{4}$ Pediatric Gastroenterology Department, HP CMNSXXI (IMSS), México

\begin{abstract}
Introduction: Neuroblastoma is relatively rare. The aim of this study was to evaluate the outcome with special focus on postoperative follow-up of a newborn with left prenatal cystic mass that evolved into lesion of mixed echogenicity with cystic and solid components.

Case Presentation: Ultrasound examination at 39 weeks of gestation revealed oligohydramnios and a cystic mass with the inability to evaluate the left fetal kidneys. Subsequently, the mother underwent an abdominal cesarean delivery of a healthy boy of $3.4 \mathrm{~kg}$. Physical examination was normal other than a large mass was palpable on upper pole of the left kidney. Postnatal ultrasound examination showed a predominantly solid suprarenal mass deforming the upper pole of the left kidney.The scan demonstrated alteration of kidney structure and displacement of the spleen and intestinal structures. The patient was admitted to an abdominal exploration with the most probable diagnosis at this stage of a cystic neuroblastoma or an adrenal hematoma as main differential diagnosis.
\end{abstract}

Results: The duration of the procedure was 240 minutes. The postoperative hospital recovery period was 20 days. No intraoperative or postoperative complications occurred. He had ultrasound control every 2 months with good report, the last control of marker tumor was normal and at 14 months after the procedure he continues with a favorable outcome.

Conclusions: The outcome of this patient was satisfactory the early detection although prenatal ultrasound and the early surgical treatment may diminish the risk of liver metastasis and chemotherapy treatment.

Keywords: Suprarenal; Neuroblastoma; Diagnosis; Surgical; Treatment; Mass; Prenatal; Pediatric; Ultrasound; Metastasis

\section{Introduction}

Masses and other anomalies may be detected in the prenatal period on second-third trimester of gestation [1,2]. In fact, Suprarenal abdominal masses in this period are often presumed to be neuroblastoma (NB), adrenal hemorrhage, messenchymal harmatoma and extra-pulmonary sequestration [2-4]. However, Neuroblastoma is relatively rare; and it is the most common adrenal-origin neoplasm and also the most common congenital malignancy accounting for approximately $30 \%$ of all fetal tumors [4,5]. Indeed, neonatal neuroblastoma represents less than $4 \%$ of all neuroblastoma cases [1]. In some countries, the neuroblastoma incidence is approximately $1 / 10,000-1 / 30,000$ live births [1,4]. Prenatal sonography is a reliable method for identifying fetal chest and abdominal masses. The location and sonographic appearance an intra-abdominal mass is important in establishing differential diagnosis [5,6]. Patient with neuroblastoma died after birth of multi-organ failure with metabolic acidosis, multi-organ hypoperfusion and disseminated coagulation induced by the metastatic disease. Generally, patients with prenatal neuroblastoma diagnosis presented liver metastasis. Hence, the location of the mass in the left adrenal gland as opposed of the more common right side [2,4]. The aim of this study was to evaluate the outcome with special focus on postoperative follow-up of a newborn with left prenatal cystic mass that evolved into 
lesion of mixed echogenicity with cystic and solid components. The procedure was performed in San Angel Inn Hospital of Chapultepec.

\section{Case Presentation}

A 40-year old woman (gravida 4, part 3, abort 1) presented at 34 weeks of gestation for her first pediatric surgery routine antenatal care visit. She had history of preeclampsia; a pelvic ultrasound was performed owing of her prenatal history a cystic mass measuring 4.6X3.6X3.4 cm was incidentally found adjacent to the upper pole of the left fetal kidney (Figure 1). Ultrasound control was performed and revealed the mass to be larger and more solid appearing. Finally, an ultrasound examination at 39 weeks of gestation revealed oligohydramnios and inability to evaluate the left fetal kidneys. Subsequently, the mother underwent an abdominal cesarean delivery of a healthy boy of $3.4 \mathrm{~kg}$. APGAR score was 9 and 10 at 1 and 5 minutes, respectively. Physical examination was normal other than a large mass was palpable on upper pole of the left kidney. Postnatal ultrasound examination showed a predominantly solid suprarenal mass deforming the upper pole of the left kidney. The scan demonstrated alteration of kidney structure and displacement of the spleen and intestinal structures (Figure 2). Serum electrolytes, complete blood count, ferritin, and transaminase and catecholamines were normal. Urinalysis and sensitivities of tumor marker levels were normal. Therefore, the patient was admitted to an abdominal exploration with the most probable diagnosis at this stage of a cystic neuroblastoma or an adrenal hematoma as main differential diagnosis.

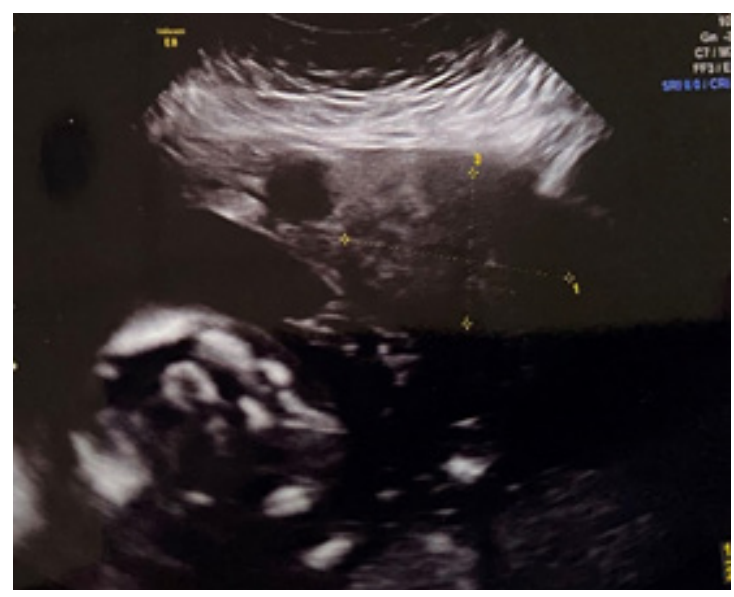

Figure 1: Prenatal sonogram with cystic mass.

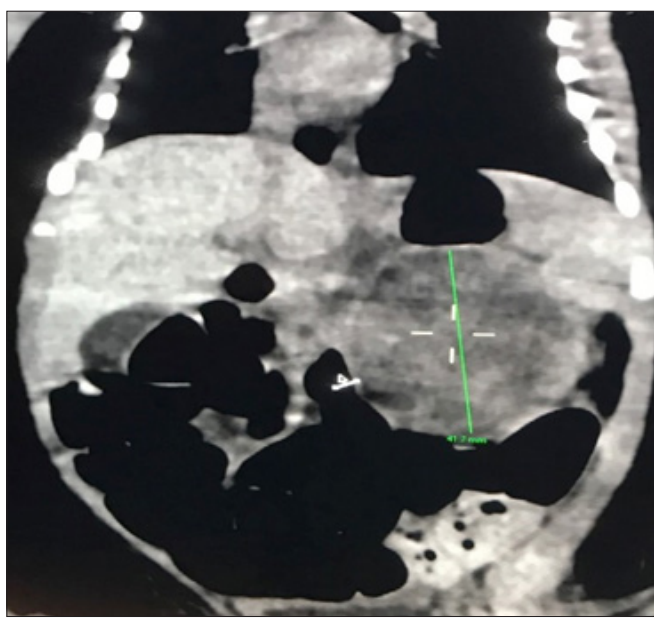

Figure 2: TC reveal complex solid cystic.

\section{Surgical Technique}

Undergo surgery; a left Middle line incision of the abdomen was performed to identify the tumor in the superior pole of the left kidney, after dissecting and moving the left colon and splenic angle of the colon. The tumor was localized and its nutritional veins were ligating from the suprarenal artery and vein. Hence, under careful ramous dissected the tumor was dislocated and exposed enough between the superior pole of the left kidney and adrenal gland from the lumbar column. Satincky clamp was placed to the part inferior of the tumor that permits the extraction of the tumor and biopsy of the suprarenal gland (Figure 3). Vein and artery of the tumor were sutured with the 6/0 PDS. Pathologic examination reveals stage 1 of neuroblastoma (Figure 4). 


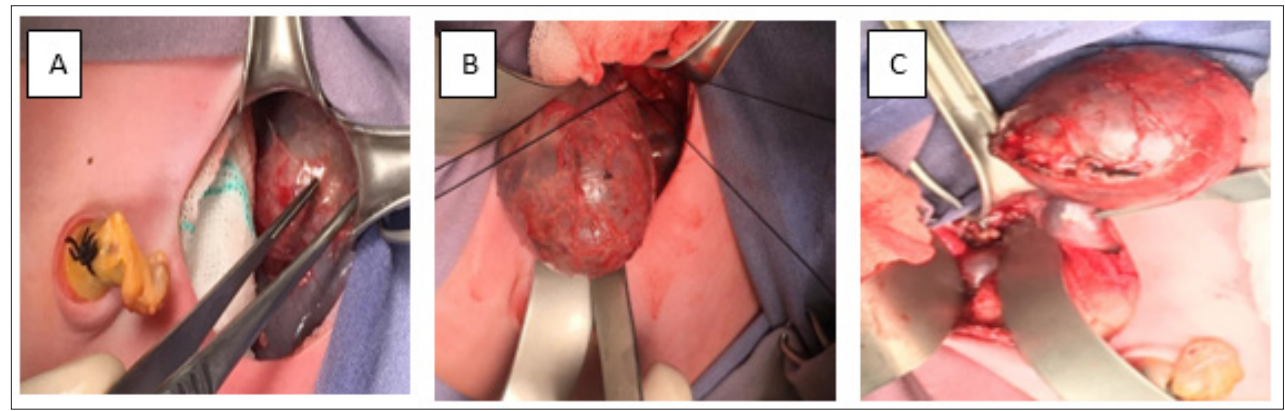

Figure 3: Congenital cystic and solid components of neuroblastoma:

A. Image shows left incision and intestinal displacement with ischemic.

B. Image demonstrates dislocation of the tumor and

C. Image shows total extraction of the tumor.
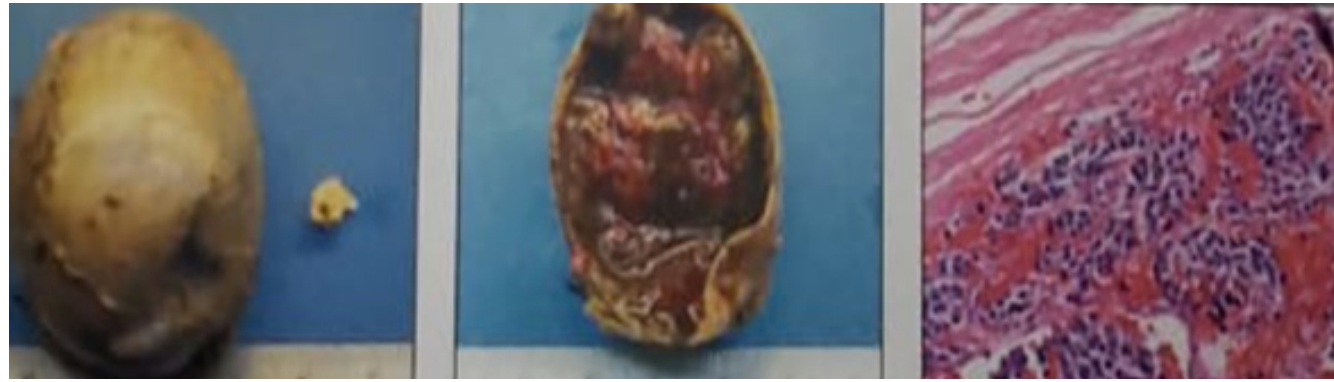

Figure 4: Pathologic study revealed neoplasm with little round cells with cystic degeneration into a solid mass with hemorrhage.

\section{Follow-ups}

The surgical time was 250 minutes with minimum blood loss and blood transfusion was not required. Middle hemodynamic changes were registered during and after the procedure. The patient presented data of necrotizing enterocolitis (NEC) and he was fasting for 5 days with parenteral nutrition, at day six of the procedure the enteral nutrition was started. The patient had a good outcome. Control abdominal ultrasound was performed, which not revealed injury of the liver and the left kidney. Furthermore, vascular complication had not reported in the Computed tomography (CT) control. The patient had antibiotic therapeutic with a third generation of cephalosporin with amikacin for seven days. Hospital stay was approximately 20 days. He had ultrasound control every 2 months with good report, the last control of marker tumor was normal and at 14 months after the procedure he continues with a favorable outcome. Under the international neuroblastoma staging system (INSS) an international neuroblastoma risk group stating system (INRG) the child had INSS stage 1, INRG L1. The histology definition based on the international neuroblastoma pathology classification (INPC) was: neoplasm with little round cells with cystic degeneration into a solid mass with hemorrhage (Figure 4).

\section{Discussion}

The vast majority of fetal masses were diagnosed in the late second and third trimesters of pregnancy, which also reflected the experience of the ultrasound (US) physician [2,7]. Generally, prenatal abdominal masses on US are characterized as cystic, solid or complex injuries as it was in this case [1,2]. In fact, $19 \%$ of the detected fetal masses occurred in the abdomen and retroperitoneal neuroblastoma represented the most common type of primary neuroblastoma at this age $[4,8]$. However, due to its rare occurrence, current evidence regarding with an early diagnosis and resection are largely extrapolated from few reports $[1,2,4,7]$. Abdominal masses involving the spinal cord that required prompt delivery and treatment before irreversible underlying organs and neurological damage occurs [1,2]. In this case the US revealed a complex mass with isoechoic image since the first trimester of the pregnancy and the post-natal US revealed $4.6 \times 3.6 \times 3.4 \mathrm{~cm}$ heterogeneous cystic mass in the left suprarenal region, which was displacing the left kidney downward and the colon to the right side with favorable form of neuroblastoma. Some authors believe that US has limitations in the characterization and diagnosis of abdominal and suprarenal masses because the findings may be nonspecific. Furthermore, abdominal and suprarenal masses in infantile period are often presumed to be NB, although cystic NB is a more commonly encountered entity than adrenal hemorrhage, mesenchymal hamartoma and extrapulmonary sequestration $[2,3,8]$.

Intravenous contrast enhanced magnetic resonance imaging (MRI) is performed for further evaluation of the lesions to avoid the ionizing radiation of computed tomography (CT) scan. Even though in this patient a CT was performed and calcification were noted in to localize mass without liver metastasis and the mass relational with 
others organs was denoted. Nearly $50 \%$ of fetal neuroblastoma is cystic, but they can also appear as solid or complex/heterogeneous masses. In fact, the mass of this case was heterogeneous with some cystic components [3-7]. Furthermore, Calcification is reported less frequent in fetal cases compared with postnatal diagnosed tumors. On the other hand, there are no current guidelines for imaging workup and follow-up of patients with the cystic variant of congenital adrenal neuroblastoma $[7,5,8]$. Furthermore, few reports observed that tumor marker assessment, have low diagnostic value in the diagnosis of cystic congenital adrenal neuroblastoma $[7,8]$. In this case the diagnosis was based on the ultrasound prenatal with postnatal control at birth and tomography at the first 48 hours of live. Neuroblastoma is the most common congenital malignancy, accounting for approximately $30 \%$ of all fetal tumors. This tumor is derived from primordial neural crest cells in the sympathetic chain ganglia and it can therefore occur anywhere along the spine from the cervical spine to the coccyx as well as in the adrenal gland [1-5].

Most fetal cases are diagnosed after 32 weeks of gestation and are usually on the right side and suprarenal. However, in this case the tumor was localized in the left side that is the main of this report with the good outcome of the patient after an early surgical procedure. On the other hand, all originated mass in the adrenal gland, nearly $50 \%$ of fetal neuroblastoma is cystic, but they can also appear as solid or complex/heterogeneous with some cystic components. In fact, this patient had a heterogeneous mass [14]. For the growing of the mass serial ultrasound examination demonstrated growth of the suprarenal mass without vertebral extension as it is reported on the literature. Although is very common, cystic NB is rare entity, which is characterized by large cystic lesions that usually originates from adrenal glands as it was in this case $[1,3,4]$. The differential diagnosis for suprarenal mass detected in a fetus include masses that are adrenal in origin neuroblastoma, asymptomatic or symptomatic adrenal hyperplasia and hemorrhage, renal duplication anomaly, origin pulmonary sequestration; enteric duplication cyst and splenic cyst [5-7]. Furthermore, the differential diagnosis between adrenal cystic neuroblastoma and adrenal hemorrhage is difficult because in few cases the value of urine catecholamine is reported normal $[4,7]$. In this patient, data of others masses were not revealed of the extension imaging pulmonary and kidney studies.

Neuroblastoma is a malignancy tumor that most commonly arises from the adrenal gland (98\%) whereas $10 \%$ of the time, it arises from the sympathetic chain two-thirds of adrenal neuroblastoma is right sided; few cases are left side as this case $[7,4]$. Furthermore, it is the unique pathologic variant that occurs in the perinatal period and it is known as cystic neuroblastoma, which is characterized by one or more macroscopic or microscopic cyst within the tumor [4]. Over time, the lesion becomes increasingly hypoechoic and typically diminishes in size with cystic degeneration and reabsorption of clot as it was observed in the pathologic report of this case [2-7]. However, definitive diagnosis of suprarenal masses using imaging studies is difficult at best. In Fact, few reports revealed that, not all suprarenal masses represent neuroblastoma; congenital mesoblastic nephroma, must also be considered $[2,5,6,8]$. Similarly of this case, cystic type of congenital adrenal neuroblastoma is limited in the literature [7]. On the other hand, the early surgical removal of congenital adrenal neuroblastoma is the most adequate treatment to provide metastasis, even though with a late diagnosis the major numbers of patients receive the surgical treatment lately $[5,8]$. In this case the diagnosis was early and the surgical removal the mass was at two days on birth, so it was early.

In few cases, it is difficult to distinct plane between the mass and the adrenal gland $[7,8]$. Hence, radical nephrectomy, renal vein lymphadenectomy, liver biopsy and bone marrow aspiration are also recommended $[7,8]$. However, despite of the compression of the tumor, it had not criteria to perform liver biopsy and radical nephrectomy in this case. Rarely, fetal neuroblastoma may metastasize to the liver or result in hydrops as it was observed in this patient $[5,6]$. In fact, pathologic examination revealed stage 1 of neuroblastoma, with favorable histologic feature. Therefore, Chemotherapy was not considered in this patient; Clinical and laboratory finding were normal after 2 weeks of the surgical treatment and tumor marker was normal, the hospital stay was tree weeks. The patient had control with US weekly and TC control at tree months of surgery without liver metastasis. Furthermore, the early detection is associated with a greater than $90 \%$ cure and metastases usually may not occur as it was in this case. However, age was found to be an independent factor associated with both long-term all-cause mortality and disease specific. In fact, the patient continues with monthly control at thirteen-months after the surgical procedure, he continued with a good follow-up. On the other hand, Univariate analysis suggested transformed age was associated with an increased risk of total death [5,7]. Usually, patients with late detection of the tumor receive 2-cycles of chemotherapy including, vincristine, etoposide and carboplatin, after the operation $[3,5,8]$. Fortunately, with the early detection by the US, the tumor was localized and resected early in its totality. Furthermore, the suggested treatment of localized tumor is surgical [5,8]. Several studies have investigated the age distribution of overall neuroblastoma and the relation between age and prognosis. In Fact, older age was associated with worse overall survival $[7,8]$. Under this relation this patient had a good prognosis.

\section{Conclusion}

Neuroblastoma is the most common adrenal malignancy in infant; the early detection although prenatal ultrasound and the early surgical treatment may diminish the risk of liver metastasis and the chemotherapy treatment is not required as it was in this case. Furthermore, in this baby we had not difficulty to perform total resection of the tumor; the outcome was very well after the surgical procedure.

\section{Acknowledgement}

A. To the San Angel Inn Hospital, Patient, Ortega Rodríguez Ma Del Carmen, Yesenia Navarro Sánchez, Jean and Reyes Families. 


\section{References}

1. Blackman SC, Evenson AR, Stephan DV, Barnewolt CE, Puder M (2008) Prenatal diagnosis and subsequent treatment of an intermediate-risk paraspinal neuroblastoma: Case report and review of the literature. Fetal Diagn Ther 24(2): 119-25.

2. Flanagan Siobhan M, Rubesova Erika, Jaramillo Diego, et al. Fetal suprarenal masses -assessing the complementary role of magnetic resonance and ultrasound for diagnosis. Pediatr Radiol 46: 246-54.

3. Aslan Mine, Deniz A, Kalyoncu AU, Habibi HA, Ozdemir GN, et al. (2017) Bilateral cystic adrenal neuroblastoma with cystic liver metastasis. APSP J Case Rep 8(1): 1.

4. Shapira G, Ilivitzki A (2015) Purely cystic adrenal lesion in a newborn evolving into a solid neuroblastoma. J Clin Ultrasound 43(2): 126-128.
5. Xiaoyan L, Jichun Z, Zhang L, Huang J, Yukui M (2020) The effect of age distribution on the prognosis of retroperitoneal neuroblastoma. Medicine (Baltimore) 99(23): e20639.

6. Rubenstein SC, Benacerraf BR, Retik AB, Mandell J (1995) Fetal suprarenal masses: Sonographic appearance and differential diagnosis. Ultrasound Obstet Gynecol 5(3): 164-167.

7. Hwang SM, Yoo SY, Kim HJ, Jeon TY (2016) Congenital Adrenal Neuroblastoma with and without cystic change: Differentiating features with an emphasis on the value of ultrasound. AJR Am J Roentgenol 207(5): 1105-1111.

8. Elizabeth S, Desai AV (2019) The evolution of risk classification for neuroblastoma. Children (Basel) 6(2): 27.

For possible submissions Click below: 